\title{
Effects of daily iron supplementation in primary-school- aged children: systematic review and meta-analysis of randomized controlled trials
}

\author{
Michael Low MBBS BMedSci, Ann Farrell MBBS, Beverley-Ann Biggs PhD, Sant-Rayn Pasricha PhD
}

See related commentary by Gray-Donald at www.cmaj.ca/lookup/doi/10.1503/cmaj.131249

\begin{abstract}
Background: Anemia is an important public health and clinical problem. Observational studies have linked iron deficiency and anemia in children with many poor outcomes, including impaired cognitive development; however, iron supplementation, a widely used preventive and therapeutic strategy, is associated with adverse effects. Primary-school-aged children are at a critical stage in intellectual development, and optimization of their cognitive performance could have long-lasting individual and population benefits. In this study, we summarize the evidence for the benefits and safety of daily iron supplementation in primaryschool-aged children.
\end{abstract}

Methods: We searched electronic databases (including MEDLINE and Embase) and other sources (July 2013) for randomized and quasirandomized controlled trials involving daily iron supplementation in children aged 5-12 years. We combined the data using random effects meta-analysis.
Results: We identified 16501 studies; of these, we evaluated 76 full-text papers and included 32 studies including 7089 children. Of the included studies, 31 were conducted in low- or middleincome settings. Iron supplementation improved global cognitive scores (standardized mean difference $0.50,95 \%$ confidence interval $[\mathrm{Cl}] 0.11$ to $0.90, p=0.01)$, intelligence quotient among anemic children (mean difference $4.55,95 \% \mathrm{Cl} 0.16$ to $8.94, p=0.04$ ) and measures of attention and concentration. Iron supplementation also improved age-adjusted height among all children and age-adjusted weight among anemic children. Iron supplementation reduced the risk of anemia by $50 \%$ and the risk of iron deficiency by $79 \%$. Adherence in the trial settings was generally high. Safety data were limited.

Interpretation: Our analysis suggests that iron supplementation safely improves hematologic and nonhematologic outcomes among primary-school-aged children in low- or middleincome settings and is well-tolerated.
$\mathrm{A}$ n estimated $25 \%$ of school-aged children worldwide are anemic. ${ }^{1}$ Iron deficiency is thought to account for about half of the global cases of anemia ${ }^{2}$ and is associated with inadequate dietary iron and, in developing settings, hookworm and schistosomiasis. ${ }^{3}$ In developed settings, anemia is prevalent among disadvantaged populations, including newly arrived refugees, indigenous people ${ }^{4}$ and some ethnic groups (e.g., Hispanic people in the United States). ${ }^{5,6}$ About 3\% of primary-school-aged children in Canada are anemic. ${ }^{7}$ Programs to address anemia are constrained by concerns that iron supplements cause adverse effects, including an increased risk of infections such as malaria in endemic areas. ${ }^{8}$

In observational studies, iron deficiency has been associated with impaired cognitive and physical development. It has been estimated that each $10 \mathrm{~g} / \mathrm{L}$ decrement in hemoglobin reduces future intelligence quotient (IQ) by 1.73 points. ${ }^{9}$ However, observational data are susceptible to confounding, ${ }^{10}$ and a causal relation between iron deficiency and cognitive impairment has not been confirmed. ${ }^{11}$ Randomized controlled trials should overcome confounding, but results of trials examining this question have not agreed.

Optimizing cognitive and physical development in primary-school-aged children could have life-long benefits. ${ }^{12}$ However, anemia-control recommendations must balance safety and efficacy. We performed a systematic review of the effects of daily iron supplementation, a commonly used strategy to combat anemia, ${ }^{2}$ in primary-schoolaged children. We examined cognitive, growth and hematologic outcomes and adverse effects across all settings.
Competing interests: Michael Low has received travel expenses from Gilead Sciences. Sant-Rayn

Pasricha is co-investigator on an unrestricted research grant from Vifor Pharma. No competing interests declared by Ann Farrell and Beverly-Ann Biggs.

This article has been peer reviewed.

Correspondence to: Sant-Rayn Pasricha, sant-rayn.pasricha@unimelb .edu.au

CMAJ 2013. DOI:10.1503 /cmaj.130628 


\section{Methods}

The protocol for our systematic review and meta-analysis is registered with PROSPERO (www.crd.york.ac.uk/PROSPERO/display_record .asp?ID=CRD42011001208). We included outcomes that could be used to inform guidelines for anemia control. ${ }^{13}$

\section{Information sources}

We searched the following electronic databases: Scopus, MEDLINE, Embase, Cochrane Central Register of Controlled Trials, ProQuest Digital Theses, the Australian Digital Theses Program database, WHO regional databases and OpenGrey. We reviewed the references of the identified articles and of previous systematic reviews. There was no language restriction applied. The search strategy for Scopus is presented in Appendix 1 (available at www.cmaj.ca/lookup /suppl/doi:10.1503/cmaj.130628/-/DC1). We performed the searches on July 4, 2013, for all databases except Embase (searched in June 2011). We searched the World Health Organization's International Clinical Trials Registry Platform in July 2013 for potentially eligible ongoing trials.

\section{Inclusion criteria}

We included randomized controlled trials that included primary-school-aged children (5-12 yr) who were randomly assigned to daily ( $\geq 5 \mathrm{~d} / \mathrm{wk}$ ) oral iron supplementation or control. We included studies that did not specifically recruit participants from this age range if the mean or median age of participants was between 5 and 12 years, if more than $75 \%$ of participants were aged 5-12 years, or if most of the study's recruitment age range overlapped 5-12 years. We excluded studies that included only children with a known developmental disability or a condition that substantially altered iron metabolism, including severe anemia. We included trials involving participants from all countries and socioeconomic backgrounds.

\section{Outcomes}

We included the following primary outcomes: cognitive performance (as measured by study authors); physical growth; safety (gastrointestinal adverse effects, infection [including malaria], morbidity and, if measured, mortality); and hemoglobin, iron indices, prevalence of anemia (hemoglobin $<120 \mathrm{~g} / \mathrm{L}$ or as defined by study authors) and iron deficiency.

The secondary outcomes were the effects of iron on other micronutrients (including zinc) and physical performance.

\section{Data extraction}

Three authors (M.L., A.F., S.-R.P.) screened the titles and abstracts identified by the search and excluded clearly ineligible references. Two authors (M.L., A.F.) independently assessed the remaining full-text studies against the inclusion criteria; their assessments were checked by a third author (S.R.P.). Data were independently extracted by 2 authors (M.L, A.F.) and checked by a third author (S.-R.P.). One author (M.L.) entered the data into Review Manager software (RevMan 5.1, The Cochrane Collaboration) and a second author (S.R.P.) checked entries for accuracy. Discrepancies were resolved through discussion.

\section{Assessment of risk of bias}

We assessed the risk of bias by use of the Cochrane risk of bias assessment tool, which addresses selection, performance, attrition, detection and reporting bias through the evaluation of reported sequence generation, allocation concealment, blinding, incomplete outcome data and selective outcome reporting. ${ }^{14}$ We used sensitivity analysis to examine the effects of removing studies with a high risk of bias (studies with poor or unclear randomization or allocation concealment, and poor or unclear blinding or high or imbalanced losses to follow-up) from the analysis. ${ }^{15}$ For outcomes that had more than 10 included trials, we examined funnel plots for evidence of publication bias.

\section{Statistical analysis}

Meta-analysis was performed for outcomes for which at least 2 studies provided data. We determined the mean difference (MD) for the difference between outcome means or the change from baseline for continuous data measured on the same scale. We calculated standardized mean differences (SMDs; difference of means is standardized according to standard deviation) for continuous data that were reported using different scales. If for a particular outcome, some studies reported outcome data only and others reported change from baseline data only, these data were combined using MD if the same scale had been used. If both outcome and change data were reported in the same study for the same outcome, the outcome data were used. For dichotomous data, the results are presented as risk ratios (RRs). For rates, the results are presented as rate ratios.

We included studies that provided iron supplementation along with a second intervention (cointervention), provided that the cointervention was applied identically in the control arm. For studies with a $2 \times 2$ factorial design (e.g., participants randomized to iron or control, then a second randomization to another intervention or 
control), we extracted the data and entered them into the meta-analysis separately.

We quantified heterogeneity using $I^{2}$. We considered substantial heterogeneity to exist if $I^{2}$ was above $50 \%$. We performed predefined subgroup analyses for outcomes that had 5 or more included studies. We included the following subgroups: baseline anemia status; baseline iron status; baseline iron deficiency anemia status; dose of iron supplementation; duration of iron supplementation; iron combination (iron alone or with a cointervention); and reported malaria endemicity of the setting. Differences between subgroups were tested using $\chi^{2}$ and $I^{2}$ statistics. ${ }^{16}$ We created forest plots to present the effect size and relative weights for each study and the overall calculated effect size. Random-effects metaanalysis was used with calculation of $\tau^{2}$ because of potential statistical, clinical and methodologic heterogeneity. ${ }^{8}$

\section{Results}

The selection of studies for inclusion is illustrated in Figure 1. Our search strategy identified 16501 potentially eligible titles and abstracts. We did not identify any potentially eligible unpublished trials in the International Clinical Trials Registry Platform. Following screening, 32 studies were deemed eligible; this included 7089 children (3837 randomized to iron supplements and 3252 to control). All studies except 1 were performed in low- or middle-income countries. Table 1 presents the characteristics of the included studies. Only 4 studies were considered at low overall risk of bias (Table 2).

The primary outcome findings are presented in Table 3. A complete table of findings including secondary outcomes is presented in Appendix 2, available at www.cmaj.ca/lookup/suppl /doi:10.1503/cmaj.130628/-/DC1.

\section{Cognition}

No studies reporting cognitive outcomes were at low overall risk of bias.

\section{Global cognitive performance}

Global cognitive performance was reported in 9 studies: 5 studies used IQ (tests: Raven Progressive Matrices, Wechsler Intelligence Scale for Children [WISC], Test of Non-verbal Intelligence 2nd Edition), ${ }^{28,43,44,55-58} 3$ studies used authoradapted scales of global cognitive performance (visual memory, digit span, mazes test, clerical task tests with maximum scores of 40), ${ }^{28-31,34}$ and 1 study used overall school performance. ${ }^{17,18}$

Data from 2 other studies could not be included because global cognitive scores were not presented.
Sen and Kanani ${ }^{52,53}$ found significant improvements from iron supplementation in digit span, maze testing and visual memory testing but not clerical tasks in 110 girls aged 9-13 years. Rico and colleagues ${ }^{47-}$ ${ }^{50}$ found no difference with iron supplementation on various cognitive domains measured with an adapted WISC in 590 children aged 6-8 years other than a small significant improvement in responses on the stimulus discrimination task.

Children who received iron supplementation had higher global cognitive scores at the end of the intervention compared with children who received the control (SMD 0.50, 95\% confidence interval [CI] 0.11 to $0.90 ; p=0.01, I^{2}=93 \%, 9$ studies, $n=2355$ ) (Figure 2). This beneficial effect was seen among children who were anemic at baseline (SMD 0.29, 95\% CI 0.07 to 0.51 ; $p=0.01, I^{2}=22 \%, 6$ studies, $n=487$ ) but not among those without anemia (SMD 0.01, 95\% CI -0.10 to $0.11 ; p=0.9, I^{2}=0 \%, 4$ studies, $n=$ 1361) (test for subgroup difference: $p=0.05$ ). Although fewer than 10 studies reported this outcome, a Funnel plot did not indicate publication bias (Appendix 3, available at www.cmaj.ca /lookup/suppl/doi:10.1503/cmaj.130628/-/DC1) .

\section{Intelligence quotient}

The most commonly reported global cognitive score was age-adjusted IQ. There was no overall

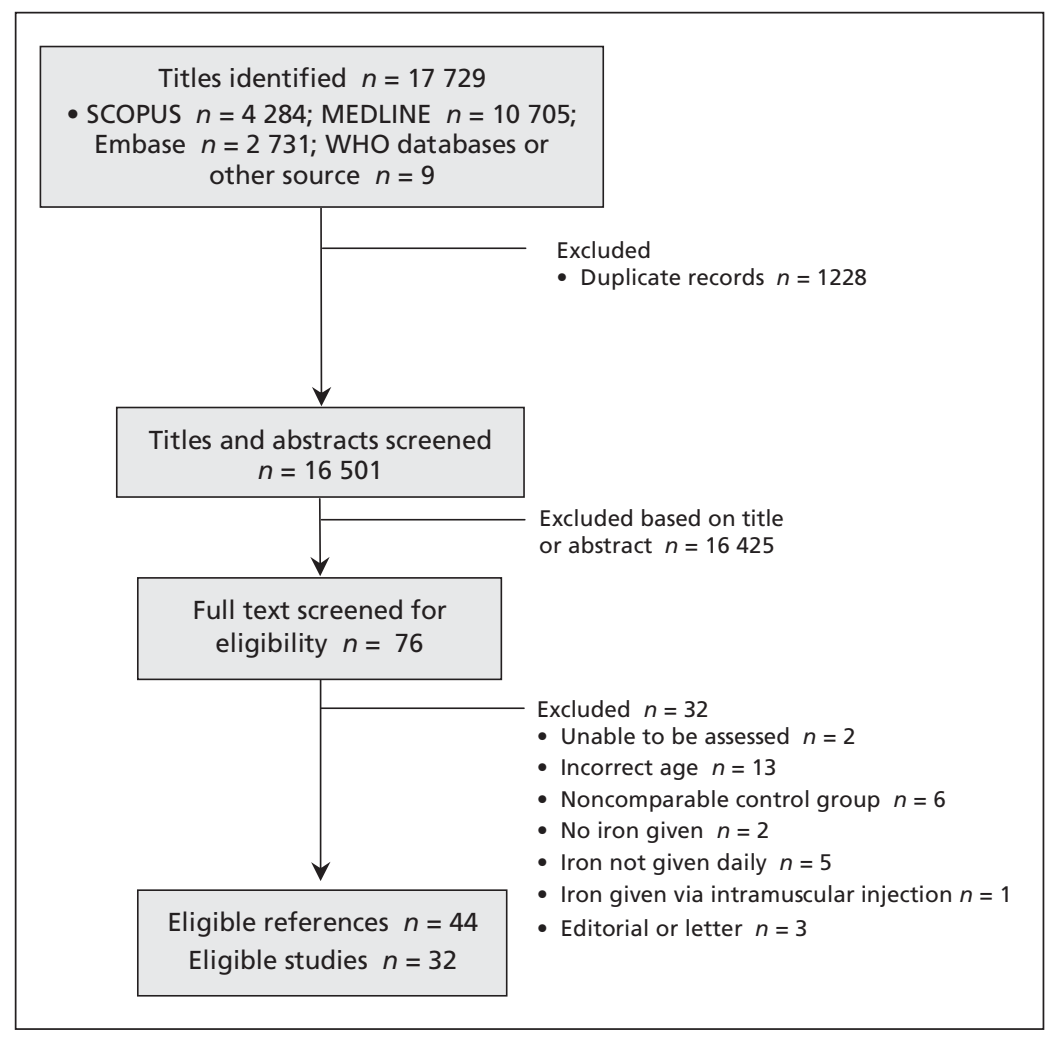

Figure 1: Flow diagram of identification of included studies. In total, 16501 titles were identified, of which 43 references pertaining to 32 studies were eligible for inclusion in the systematic review. 
Table 1 (part 1 of 3): Studies included in the systematic review*

\begin{tabular}{|c|c|c|c|c|c|c|c|c|}
\hline Study & Country & $\begin{array}{c}\text { Age at } \\
\text { recruitment, } \\
y r\end{array}$ & $\begin{array}{l}\text { Baseline anemia, } \\
\text { iron deficiency status }\end{array}$ & Intervention & Control & No. randomized & Duration & Outcomes \\
\hline Ayoya et al. ${ }^{17,18}$ & Mali & $7-12$ & $\begin{array}{l}\text { Anemic, iron status } \\
\text { unknown }\end{array}$ & $\begin{array}{l}\text { Ferrous sulfate } \\
100 \mathrm{mg}+ \\
\text { anthelmintic } \\
\text { therapy }\end{array}$ & $\begin{array}{l}\text { Anthelmintic } \\
\text { therapy }\end{array}$ & $\begin{array}{l}\text { Total: 202; iron: } \\
\text { 105; placebo: } 97\end{array}$ & $12 \mathrm{wk}$ & $\begin{array}{l}\text { Anemia, hemoglobin, } \\
\text { malaria, ferritin, } \\
\text { soluble transferrin } \\
\text { receptor, cognitive } \\
\text { score }\end{array}$ \\
\hline Boivin et al. ${ }^{20}$ & Zaire & $\begin{array}{l}\text { Mean age } \\
7.7\end{array}$ & $\begin{array}{l}\text { Anemia, iron status } \\
\text { unknown }\end{array}$ & $\begin{array}{l}20 \mathrm{~mL} \text { of iron } \\
\text { supplementation } \\
\text { (formulation not } \\
\text { stated) } \pm \\
\text { levamisole }\end{array}$ & $\begin{array}{l}\text { No } \\
\text { intervention } \\
\pm \text { levamisole }\end{array}$ & $\begin{array}{l}\text { Total: 47; iron: 7; } \\
\text { placebo: } 15 ; \text { iron } \\
\text { + anthelmintic: } \\
\text { 17; placebo + } \\
\text { levamisole: } 8\end{array}$ & $\begin{array}{l}4 \mathrm{wk} \\
30 \mathrm{~d}\end{array}$ & $\begin{array}{l}\text { Data not extractable } \\
\text { (all data presented as } \\
\text { levamisole v. no } \\
\text { levamisole) (not } \\
\text { included in analysis) }\end{array}$ \\
\hline Charoenlarp 22 & Thailand & $5-14$ & $\begin{array}{l}\text { Anemia and iron } \\
\text { status unknown }\end{array}$ & $\begin{array}{l}\text { Ferrous gluconate } \\
(300 \mathrm{mg}) \pm \text { folate }\end{array}$ & $\begin{array}{l}\text { Placebo } \\
\pm \text { folate }\end{array}$ & $\begin{array}{l}\text { Total: 437; iron: } \\
\text { 214; placebo: } \\
223\end{array}$ & $3 \mathrm{mo}$ & Hematocrit \\
\hline $\begin{array}{l}\text { Charoenlarp } \\
\text { et al. } .^{23}\end{array}$ & Thailand & $6-12$ & $\begin{array}{l}\text { Anemia and iron } \\
\text { status unknown }\end{array}$ & $\begin{array}{l}\text { Ferrous sulfate } \\
(200 \mathrm{mg}) \pm \\
\text { mebendazole } \\
\text { (if hookworms } \\
\text { present) }\end{array}$ & $\begin{array}{l}\text { Placebo } \pm \\
\text { mebendazole } \\
\text { (if hook- } \\
\text { worms } \\
\text { present) }\end{array}$ & $\begin{array}{l}\text { Total: 72; iron: } \\
\text { 33; placebo: } 39\end{array}$ & $5 \mathrm{mo}$ & $\begin{array}{l}\text { Hemoglobin, } \\
\text { hematocrit }\end{array}$ \\
\hline Chwang et al. ${ }^{24}$ & Indonesia & $8.2-13.5$ & $\begin{array}{l}\text { Subgroups: anemic, } \\
\text { not anemic, iron } \\
\text { deficient, not iron } \\
\text { deficient, iron } \\
\text { deficiency anemia, } \\
\text { nonanemic noniron } \\
\text { deficient }\end{array}$ & $\begin{array}{l}\text { Ferrous sulfate } \\
(10 \mathrm{mg})\end{array}$ & Placebo & $\begin{array}{l}\text { Total: } 139 \text {; iron: } \\
\text { 59; placebo: } 60\end{array}$ & $12 \mathrm{wk}$ & $\begin{array}{l}\text { Hemoglobin, serum } \\
\text { iron, transferrin } \\
\text { saturation, total iron } \\
\text { binding capacity, } \\
\text { height, weight, arm } \\
\text { circumference, } \\
\text { morbidity score; z } \\
\text { scores for height, } \\
\text { weight, arm } \\
\text { circumference }\end{array}$ \\
\hline Fallahi et al. ${ }^{26}$ & Iran & $\begin{array}{l}\text { Grade } 5 \\
\text { (about } \\
\text { age } 11 \mathrm{yr} \text { ) }\end{array}$ & $\begin{array}{l}\text { Anemia and iron } \\
\text { status unknown }\end{array}$ & $\begin{array}{l}\text { Ferrous sulfate } \\
(20 \mathrm{mg})+\text { zinc }\end{array}$ & Zinc & $\begin{array}{l}\text { Total: 54; iron: } \\
\text { 26; placebo: } 28\end{array}$ & $4 \mathrm{mo}$ & $\begin{array}{l}\text { Hemoglobin, ferritin, } \\
\text { zinc, retinol }\end{array}$ \\
\hline Gebreselassie $^{27}$ & Ethiopia & $5-14$ & $\begin{array}{l}\text { Anemic and iron } \\
\text { deficient }\end{array}$ & $\begin{array}{l}\text { Ferrous sulfate } \\
(300 \mathrm{mg})\end{array}$ & Placebo & $\begin{array}{l}\text { Total: } 480 \text {; iron: } \\
\text { 239; placebo: } \\
241\end{array}$ & $3 \mathrm{mo}$ & $\begin{array}{l}\text { Anemia, iron } \\
\text { deficiency, } \\
\text { hemoglobin, ferritin, } \\
\text { malaria, splenomegaly }\end{array}$ \\
\hline $\begin{array}{l}\text { Gopaldas } \\
\text { et al. } \text {. }^{28-31}\end{array}$ & India & $8-15$ & $\begin{array}{l}\text { Iron status unknown. } \\
\text { Subgroups: anemic, } \\
\text { nonanemic }\end{array}$ & $\begin{array}{l}\text { Ferrous sulfate } \\
(30 \mathrm{mg} \text { or } 40 \mathrm{mg})\end{array}$ & Placebo & $\begin{array}{l}\text { Total: 210; iron: } \\
\text { 140; placebo: } 70\end{array}$ & $60 d$ & $\begin{array}{l}\text { Hemoglobin, cognitive } \\
\text { scores, exercise tests }\end{array}$ \\
\hline Greisen $^{32}$ & Zambia & $5-15$ & $\begin{array}{l}\text { Anemic, iron status } \\
\text { unknown }\end{array}$ & $\begin{array}{l}\text { Ferrous fumarate } \\
(300 \mathrm{mg}) \pm \text { folate }\end{array}$ & $\begin{array}{l}\text { Placebo } \\
\pm \text { folate }\end{array}$ & $\begin{array}{l}\text { Total: } 430 \text {; iron: } \\
218 ; \text { placebo: } \\
212\end{array}$ & $7 \mathrm{wk}$ & Hemoglobin \\
\hline Harvey et al. ${ }^{33}$ & $\begin{array}{l}\text { Papua New } \\
\text { Guinea }\end{array}$ & $8-12$ & $\begin{array}{l}\text { Anemic, iron status } \\
\text { unknown }\end{array}$ & $\begin{array}{l}\text { Ferrous sulfate } \\
(200 \mathrm{mg})\end{array}$ & Placebo & $\begin{array}{l}\text { Total: } 300 \text {; iron: } \\
\text { 156; placebo: } \\
144\end{array}$ & $16 \mathrm{wk}$ & $\begin{array}{l}\text { Hemoglobin, gastro- } \\
\text { intestinal upset, } \\
\text { malaria }\end{array}$ \\
\hline
\end{tabular}


Table 1 (part 2 of 3): Studies included in the systematic review*

\begin{tabular}{|c|c|c|c|c|c|c|c|c|}
\hline Study & Country & $\begin{array}{l}\text { Age at } \\
\text { recruitment, } \\
\text { yr }\end{array}$ & $\begin{array}{l}\text { Baseline anemia, } \\
\text { iron deficiency status }\end{array}$ & Intervention & Control & No. randomized & Duration & Outcomes \\
\hline Kashyap et al. ${ }^{34}$ & India & $8-15$ & $\begin{array}{l}\text { Anemia and iron } \\
\text { status unknown }\end{array}$ & $\begin{array}{l}\text { Ferrous sulfate } \\
(200 \mathrm{mg})\end{array}$ & Placebo & $\begin{array}{l}\text { Total: 130; iron: } \\
65 ; \text { placebo: } 65\end{array}$ & $8 \mathrm{mo}$ & $\begin{array}{l}\text { Hemoglobin, cognitive } \\
\text { scores }\end{array}$ \\
\hline Konofal et al. ${ }^{35}$ & France & $5-8$ & $\begin{array}{l}\text { Anemia status } \\
\text { unknown, iron } \\
\text { deficient }\end{array}$ & $\begin{array}{l}\text { Ferrous sulfate } \\
(300 \mathrm{mg})\end{array}$ & Placebo & $\begin{array}{l}\text { Total: 23; iron: } \\
\text { 18; placebo: } 5\end{array}$ & $3 \mathrm{mo}$ & $\begin{array}{l}\text { Hemoglobin, ferritin, } \\
\text { serum iron, transferrin } \\
\text { saturation, } \\
\text { constipation and } \\
\text { abdominal pain }\end{array}$ \\
\hline Latham et al. ${ }^{36}$ & Kenya & $\begin{array}{l}\text { Mean age } \\
\text { control: } \\
8.1 \text {; iron: } \\
7.9\end{array}$ & $\begin{array}{l}\text { Anemia and iron } \\
\text { status unknown }\end{array}$ & $\begin{array}{l}\text { Ferrous sulfate } \\
(400 \mathrm{mg})+ \\
\text { albendazole }\end{array}$ & $\begin{array}{l}\text { Placebo + } \\
\text { albendazole }\end{array}$ & $\begin{array}{l}\text { Total: 55; iron: } \\
\text { 29; placebo: } 26\end{array}$ & 15 wk & $\begin{array}{l}\text { Hemoglobin, height, } \\
\text { weight, arm circum- } \\
\text { ference, malaria, } \\
\text { Ascaris infection }\end{array}$ \\
\hline Lawless et al. ${ }^{37}$ & Kenya & $6-11$ & $\begin{array}{l}\text { Anemia and iron } \\
\text { status unknown }\end{array}$ & $\begin{array}{l}\text { Ferrous sulfate } \\
(150 \mathrm{mg})\end{array}$ & Placebo & $\begin{array}{l}\text { Total: 86; iron: } \\
\text { 42; placebo: } 44\end{array}$ & $14 \mathrm{wk}$ & $\begin{array}{l}\text { Hemoglobin, ferritin, } \\
\text { height, weight, z scores } \\
\text { for height and weight }\end{array}$ \\
\hline Nchito et al. ${ }^{38-40}$ & Zambia & $\begin{array}{l}7-15 \\
\text { (mean 10.2) }\end{array}$ & $\begin{array}{l}\text { Anemia and iron } \\
\text { status unknown }\end{array}$ & $\begin{array}{l}\text { Ferrous dextran } \\
\text { ( } 60 \mathrm{mg} \text { elemental } \\
\text { iron }) \pm \text { multi- } \\
\text { vitamin }\end{array}$ & $\begin{array}{l}\text { Placebo } \pm \\
\text { multivitamin }\end{array}$ & $\begin{array}{l}\text { Total: } 215 \text {; iron: } \\
61 ; \text { placebo: } 50 ; \\
\text { Iron + multi- } \\
\text { vitamin: } 57 \text {; } \\
\text { placebo + multi- } \\
\text { vitamin: } 47\end{array}$ & $10 \mathrm{mo}$ & Ascaris infection \\
\hline Paracha et al. ${ }^{41}$ & Pakistan & 11 & $\begin{array}{l}\text { Subgroups: anemic, } \\
\text { not anemic, iron } \\
\text { deficient, not iron } \\
\text { deficient, iron } \\
\text { deficient anemia, } \\
\text { nonanemic noniron } \\
\text { deficient }\end{array}$ & $\begin{array}{l}\text { Ferrous gluconate } \\
\text { (76 mg elemental } \\
\text { iron) + } \\
\text { multivitamin }\end{array}$ & $\begin{array}{l}\text { Placebo + } \\
\text { multivitamin }\end{array}$ & $\begin{array}{l}\text { Total: 119; iron: } \\
61 ; \text { placebo: } 58\end{array}$ & $11 \mathrm{wk}$ & $\begin{array}{l}\text { Hemoglobin, } \\
\text { hematocrit, MCV, MCH, } \\
\text { MCHC, ferritin, } \\
\text { transferrin saturation }\end{array}$ \\
\hline Perrone et al. ${ }^{42}$ & Italy & $4-11$ & $\begin{array}{l}\text { Not anemic, iron } \\
\text { status unknown }\end{array}$ & $\begin{array}{l}\text { Iron polymaltose } \\
\text { ( } 12.5 \mathrm{mg} \\
\text { elemental iron) }\end{array}$ & Placebo & $\begin{array}{l}\text { Total: } 16 \text {; iron: } 8 ; \\
\text { placebo: } 8\end{array}$ & $1 \mathrm{yr}$ & $\begin{array}{l}\text { Ferritin, zinc, serum } \\
\text { copper, z scores for } \\
\text { weight and height }\end{array}$ \\
\hline Pollitt et al. ${ }^{43,44}$ & Thailand & $9-11$ & $\begin{array}{l}\text { Subgroups: anemic, } \\
\text { not anemic, iron } \\
\text { deficient, not iron } \\
\text { deficient, iron } \\
\text { deficient anemia, } \\
\text { iron deficient not } \\
\text { anemic, nonanemic } \\
\text { noniron deficient }\end{array}$ & $\begin{array}{l}\text { Ferrous sulfate } \\
\text { ( } 50 \mathrm{mg} / 100 \mathrm{mg} \text { ) } \\
\text { + albendazole }\end{array}$ & $\begin{array}{l}\text { Placebo + } \\
\text { albendazole }\end{array}$ & $\begin{array}{l}\text { Total: 1358; iron: } \\
679 ; \text { placebo: } \\
679\end{array}$ & $16 \mathrm{wk}$ & $\begin{array}{l}\text { Hemoglobin, ferritin, } \\
\text { transferrin saturation, } \\
\text { cognitive scores }\end{array}$ \\
\hline Powers et al. ${ }^{45}$ & Gambia & $4-12$ & $\begin{array}{l}\text { Anemia and iron } \\
\text { status unknown }\end{array}$ & $\begin{array}{l}\text { Ferrous sulfate } \\
(200 \mathrm{mg})\end{array}$ & Placebo & $\begin{array}{l}\text { Total: 50; iron: } \\
\text { 25; placebo: } 25\end{array}$ & $6 \mathrm{wk}$ & $\begin{array}{l}\text { Hemoglobin, } \\
\text { hematocrit, } \mathrm{MCV}, \mathrm{MCH}, \\
\text { serum iron }\end{array}$ \\
\hline Richard et al. ${ }^{46}$ & Peru & $0.5-15$ & $\begin{array}{l}\text { Anemia and iron } \\
\text { status unknown }\end{array}$ & $\begin{array}{l}\text { Ferrous sulfate } \\
(15 \mathrm{mg} \text { elemental } \\
\text { iron) } \pm \text { zinc }\end{array}$ & $\begin{array}{l}\text { Placebo } \\
\pm \text { zinc }\end{array}$ & $\begin{array}{l}\text { Total: 836; iron: } \\
\text { 208; placebo: } \\
\text { 209; iron + zinc: } \\
\text { 210; placebo + } \\
\text { zinc: } 209\end{array}$ & $7 \mathrm{mo}$ & $\begin{array}{l}\text { Hemoglobin, zinc, } \\
\text { malaria, lower } \\
\text { respiratory tract } \\
\text { infection, diarrhea }\end{array}$ \\
\hline Rico et al. ${ }^{47-50}$ & Mexico & $6-8$ & $\begin{array}{l}\text { Anemia and iron } \\
\text { status unknown }\end{array}$ & $\begin{array}{l}\text { Ferrous fumarate } \\
\text { (10 mg elemental } \\
\text { iron) } \pm \text { zinc }\end{array}$ & $\begin{array}{l}\text { Placebo } \\
\text { or zinc }\end{array}$ & $\begin{array}{l}\text { Total: 590; iron: } \\
\text { 141; placebo: } \\
\text { 151; iron + zinc: } \\
\text { 152; placebo + } \\
\text { zinc: } 146\end{array}$ & $6 \mathrm{mo}$ & $\begin{array}{l}\text { Hemoglobin, ferritin, } \\
\text { zinc, lead, height, } \\
\text { weight, cognitive } \\
\text { scores }\end{array}$ \\
\hline Rosales et al. ${ }^{51}$ & Guatemala & $8-11$ & $\begin{array}{l}\text { Anemia and iron } \\
\text { status unknown }\end{array}$ & $\begin{array}{l}\text { Iron citrate } \\
(20 \mathrm{mg} \text { elemental } \\
\text { iron) } \pm \text { zinc }\end{array}$ & $\begin{array}{l}\text { Placebo } \\
\pm \text { zinc }\end{array}$ & $\begin{array}{l}\text { Total: } 64 \text {; iron: } \\
\text { 15; placebo: } 15 ; \\
\text { iron + zinc: } 19 ; \\
\text { placebo + zinc: } \\
15\end{array}$ & $8 w k$ & $\begin{array}{l}\text { Iron deficiency, zinc } \\
\text { deficiency, } \\
\text { hemoglobin, ferritin, } \\
\text { zinc, CRP }\end{array}$ \\
\hline Sen et al. ${ }^{52,53}$ & India & $9-13$ & $\begin{array}{l}\text { Anemia and iron } \\
\text { status unknown }\end{array}$ & $\begin{array}{l}\text { Unknown (100 mg } \\
\text { elemental iron) } \\
+ \text { folic acid }\end{array}$ & Control & $\begin{array}{l}\text { Total: } 110 \text {; iron: } \\
\text { 59; placebo: } 41\end{array}$ & $1 \mathrm{yr}$ & $\begin{array}{l}\text { Hemoglobin, cognitive } \\
\text { scores, maximum steps } \\
\text { climbed, recovery time }\end{array}$ \\
\hline \multirow[t]{2}{*}{ Sen et al..$^{54}$} & India & $9-13$ & $\begin{array}{l}\text { Iron status unknown. } \\
\text { Subgroups: anemic, } \\
\text { not anemic }\end{array}$ & $\begin{array}{l}\text { Unknown } \\
\text { (elemental iron } \\
100 \mathrm{mg} \text { ) }\end{array}$ & Control & $\begin{array}{l}\text { Total: } 100 ; \text { iron: } \\
\text { 59; placebo: } 41\end{array}$ & $1 \mathrm{yr}$ & Hemoglobin \\
\hline & & & & & & & & Continued \\
\hline
\end{tabular}




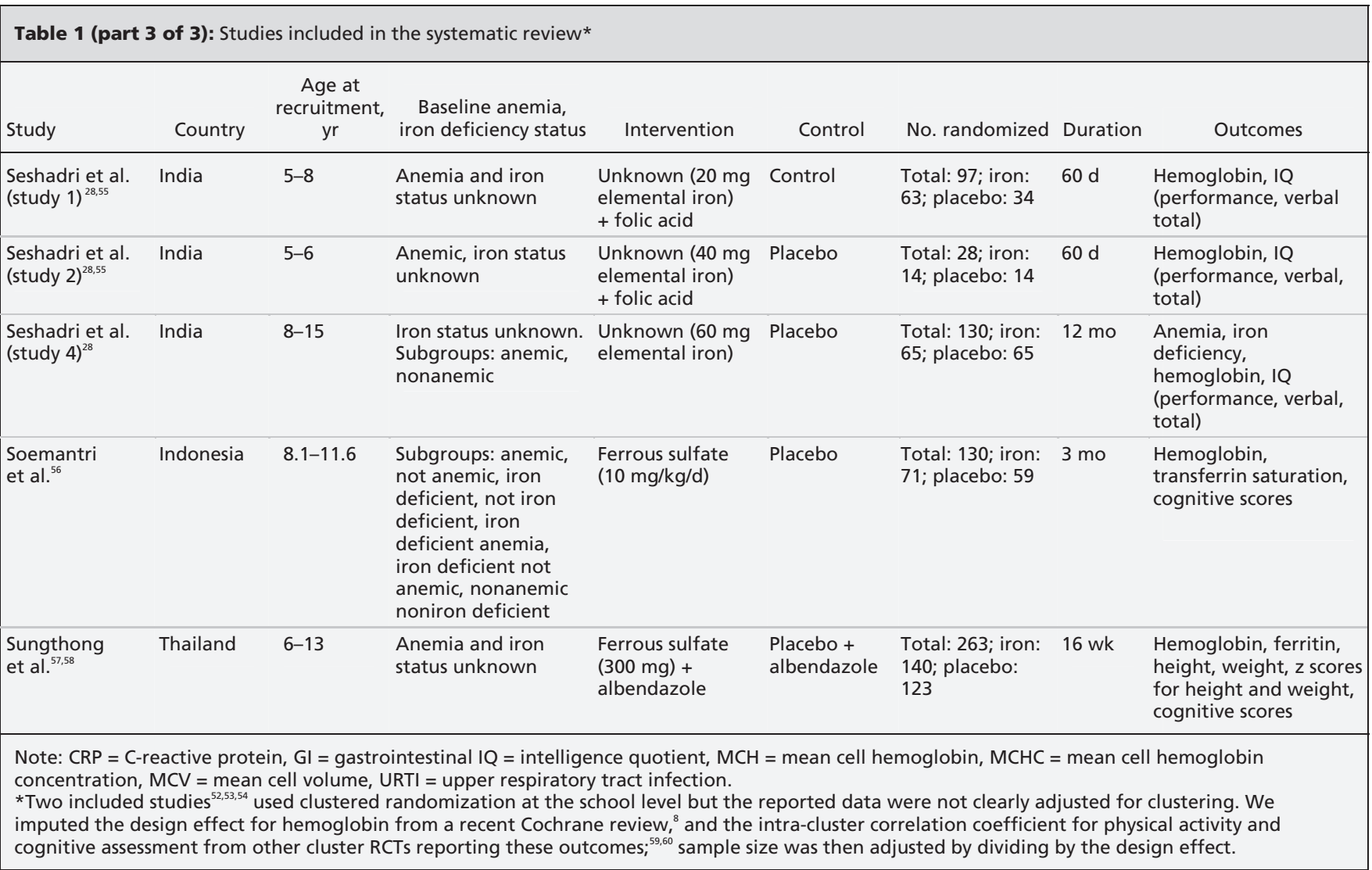

benefit of iron supplementation on IQ scores (MD 5.47, 95\% CI -3.24 to $4.18 ; p=0.2, I^{2}=$ $97 \%, 5$ studies, $n=1874$ ). However, our subgroup analysis showed that children who had anemia at baseline had significant improvements in their IQ after iron supplementation compared with children who received the control (MD $4.55,95 \%$ CI 0.16 to $8.94 ; p=0.04, I^{2}=28 \%, 3$ studies, $n=186$ ) (Appendix 3).

\section{Other cognitive tests}

Compared with children in the control group, children who received iron supplementation had superior performance on the maze test (MD $1.30,95 \%$ CI 0.9 to $1.7 ; p<0.001, I^{2}=0 \%, 4$ studies, $n=288$ ) and clerical task scores (SMD $0.44,95 \%$ CI 0.14 to $0.75 ; p=0.005, I^{2}=30 \%, 4$ studies, $n=290$ ). There was no difference between iron supplementation and control for other measures including digit span, visual memory score, math scores and language test scores (Appendix 2).

\section{Growth}

\section{Height}

Iron supplementation was not associated with a difference in absolute height. However, children who received iron supplements experienced a small advantage in height-for-age (z score) (Figure 3 ). When we included only studies with a low risk of bias, the benefit of iron supplementation was still apparent (MD 0.12, 95\% CI 0.00 to 0.23; $p=0.05$, 2 studies, $n=922$ ).

\section{Weight}

There was no difference in absolute weight or weight-for-age (z score) between children who received iron or control. One study included subgroups of children with iron deficiency anemia and children without iron deficiency or anemia. ${ }^{24}$ The authors reported that the children with iron deficiency anemia who received iron had a significant improvement in weight-for-age (MD $0.13,95 \%$ CI 0.04 to $0.22 ; p=0.005, n=78$ ); this effect was not seen among children without anemia (MD $0.18,95 \%$ CI -0.24 to $0.60 ; p=$ $0.4, n=57) .^{24}$

\section{Safety}

\section{Malaria infection}

The prevalence of malaria parasitemia was similar between iron and control groups (RR 1.10, $95 \%$ CI 0.94 to $1.29 ; p=0.9, I^{2}=93 \%, 4$ studies, $n=919$ ) (Appendix 4, available at www.cmaj.ca /lookup/suppl/doi:10.1503/cmaj.130628/-/DC1). There were no differences in the rate of positive 
tests for all malaria infections (rate ratio 1.05, 95\% CI 0.90 to $1.21 ; p=0.6, I^{2}=0 \%, 4$ studies, $n=1818)$, Plasmodium falciparum infections (rate ratio $1.02,95 \% \mathrm{CI} 0.81$ to $1.29 ; p=0.9, I^{2}$ $=0 \%, 2$ studies, $n=1136)$ and Plasmodium vivax infections (rate ratio $1.07,95 \% \mathrm{CI} 0.85$ to $1.34 ; p=0.6, I^{2}=0 \%, 2$ studies, $n=1136$ ).

\section{Other infections}

De Silva and colleagues ${ }^{25}$ reported fewer respiratory tract infections (MD $-0.56,95 \% \mathrm{CI}-0.82$ to $-0.30 ; p<0.001, n=363$ ) and fewer days off sick with respiratory tract infections (MD -7.04 ,
95\% CI -9.75 to $-4.33 ; p<0.001, n=363$ ) among children who received iron supplementation. Richard and colleagues ${ }^{46}$ reported no difference in the rate of acute respiratory tract infections between groups (rate ratio $0.91,95 \% \mathrm{CI}$ $0.65-1.27 ; p=0.6, n=836)$.

\section{Gastrointestinal adverse effects}

There was no difference in the number of children with gastrointestinal upset, constipation, vomiting or diarrhea or the rates of diarrhea between children who received iron supplementation or control (Appendix 4).

Table 2: Analysis of the risk of bias among the included studies

\begin{tabular}{|c|c|c|c|c|c|c|}
\hline Study & $\begin{array}{c}\text { Random } \\
\text { sequence } \\
\text { generation } \\
\text { (selection bias) }\end{array}$ & $\begin{array}{c}\text { Allocation } \\
\text { concealment } \\
\text { (selection bias) }\end{array}$ & $\begin{array}{l}\text { Blinding } \\
\text { (participants and } \\
\text { personnel; } \\
\text { performance bias) }\end{array}$ & $\begin{array}{c}\text { Blinding } \\
\text { (outcome } \\
\text { assessment; } \\
\text { detection bias) }\end{array}$ & $\begin{array}{l}\text { Incomplete } \\
\text { outcome data } \\
\text { (attrition bias) }\end{array}$ & $\begin{array}{l}\text { Selective } \\
\text { reporting } \\
\text { (reporting bias) }\end{array}$ \\
\hline Ayoya et al. ${ }^{17,18}$ & Yes & Yes & Yes & Yes & Yes & Unknown \\
\hline Berger et al. ${ }^{19}$ & Unknown & Unknown & Yes & Yes & Yes & Unknown \\
\hline Boivin et al. ${ }^{20}$ & Unknown & Unknown & Unknown & Unknown & Yes & Unknown \\
\hline Charoenlarp $\mathrm{p}^{22}$ & Unknown & Unknown & Yes & Yes & Yes & Unknown \\
\hline Charoenlarp et al. ${ }^{23}$ & Unknown & Unknown & Yes & Yes & Unknown & Unknown \\
\hline Chwang et al. ${ }^{24}$ & Unknown & Unknown & Unknown & Yes & Unknown & Unknown \\
\hline de Silva et al. ${ }^{25}$ & Unknown & Unknown & Yes & Yes & Yes & Unknown \\
\hline Fallahi et al. $^{26}$ & Unknown & Unknown & Yes & Yes & Yes & Unknown \\
\hline Harvey et al. ${ }^{33}$ & Unknown & Unknown & Unknown & Unknown & Yes & Unknown \\
\hline Kashyap et al. ${ }^{34}$ & Unknown & Unknown & Unknown & Unknown & Yes & Unknown \\
\hline Konofal et al. ${ }^{35}$ & Yes & Unknown & Yes & Yes & Yes & Unknown \\
\hline Latham et al. ${ }^{36}$ & Unknown & Unknown & Unknown & Yes & Yes & Unknown \\
\hline Lawless et al. ${ }^{37}$ & Yes & Yes & Yes & Yes & Unknown & Unknown \\
\hline Nchito et al. ${ }^{38-40}$ & Unknown & No & Unknown & Yes & No & Unknown \\
\hline Paracha et al. ${ }^{41}$ & Unknown & Unknown & Unknown & Yes & Yes & Unknown \\
\hline Perrone et al. ${ }^{42}$ & Unknown & Unknown & Unknown & Yes & Yes & Unknown \\
\hline Pollitt et al. ${ }^{43,44}$ & Unknown & Unknown & Yes & Yes & No & No \\
\hline Seshadri et al. (study 1$)^{28,55}$ & Unknown & Unknown & No & No & Unknown & Unknown \\
\hline Seshadri et al. (study 2 ) 28,55 & Yes & Unknown & Unknown & Unknown & Unknown & Unknown \\
\hline Seshadri et al. (study 4$)^{28}$ & Unknown & Unknown & Unknown & Unknown & Unknown & Unknown \\
\hline Soemantri et al. ${ }^{56}$ & Unknown & Unknown & Unknown & Unknown & Unknown & Unknown \\
\hline Sungthong et al. ${ }^{57,58}$ & Yes & Yes & Unknown & Unknown & Yes & Unknown \\
\hline
\end{tabular}


Morbidity and mortality

Chwang and colleagues ${ }^{24}$ reported that children who received iron supplementation had a lower overall morbidity score than children in the control group (MD $-2.73,95 \% \mathrm{CI}-3.56$ to -1.90 ; $p<0.001, n=119)$. Mortality, admission to hospital, nonregular clinic visits and morbidity from iron poisoning were not reported in any study.

\section{Hematologic and iron indices}

Iron supplementation improved hemoglobin and ferritin levels, anemia and iron deficiency (Table 3, Appendix 2, Appendix 5 [available at www .cmaj.ca/lookup/suppl/doi:10.1503/cmaj.130628 /-/DC1]). Funnel plots for hemoglobin and ferritin levels did not show any evidence of publication bias (Appendix 5). When we included only the 4 studies at low risk of bias, the beneficial effects of iron supplementation were no longer observed for hemoglobin, ferritin or iron deficiency.

Only 2 studies reported iron deficiency anemia. ${ }^{17,18,57,58}$ Iron supplementation reduced the prevalence of anemia (RR $0.12,95 \%$ CI 0.02 to $0.66 ; p=0.01,334$ children).

\section{Other micronutrients}

There were no differences in the prevalence of zinc deficiency ${ }^{51}$ or in the serum levels of zinc, ${ }^{51}$ lead, ${ }^{47-50}$ retinol $^{26}$ or copper. ${ }^{42}$

\section{Exercise performance}

Gopaldas and colleagues ${ }^{28-31}$ reported a reduction in pulse rate during the Harvard Step Test among children who received iron supplementation. However, Sen and Kanani ${ }^{52,53}$ found no difference between the iron and control groups in the number of steps climbed or recovery time.

\section{Adherence}

Twenty-one studies reported adherence. We were unable, however, to perform a meta-analysis because of differences in reporting. Five studies (835 children) used fully or partially supervised iron supplementation. Of the remaining studies, 4 (820 children) reported adherence of more than $90 \%$ in the control and iron supplementation groups, ${ }^{21,25,27,57,58}$ and 7 studies (2071 children) reported more than $80 \%$ compliance in both groups. ${ }^{19,21,25,27,3,3,46,57,58}$ Only 3 studies reported lower

Table 3: Effect of iron supplementation on primary outcomes among children aged 5-12 years

\begin{tabular}{|c|c|c|c|c|c|}
\hline Outcome & $\begin{array}{l}\text { No. of } \\
\text { trials }\end{array}$ & $\begin{array}{c}\text { No. of } \\
\text { participants }\end{array}$ & Effect size $(95 \% \mathrm{Cl})$ & $p$ for effect & $I^{2}, \%$ \\
\hline \multicolumn{6}{|l|}{ Cognitive performance } \\
\hline Global cognitive performance* & 9 & 2355 & 0.50 (0.11 to 0.90$)$ & 0.01 & 93 \\
\hline Intelligence quotient $\dagger$ & 5 & 1874 & $5.47(-3.24$ to 14.18$)$ & 0.2 & 97 \\
\hline \multicolumn{6}{|l|}{ Growth } \\
\hline Height† & 5 & 1111 & $-0.37(-1.14$ to 0.40$)$ & 0.3 & 0 \\
\hline Z score for height $\dagger$ & 5 & 1318 & 0.09 (0.01 to 0.17$)$ & 0.03 & 10 \\
\hline Weight $†$ & 5 & 1111 & $0.12(-0.50$ to 0.75$)$ & 0.7 & 0 \\
\hline Z score for weight $\dagger$ & 5 & 1318 & $0.10(-0.03$ to 0.23$)$ & 0.1 & 70 \\
\hline \multicolumn{6}{|l|}{ Hematology and iron indices } \\
\hline Hemoglobin, g/L $\dagger$ & 28 & 6545 & $8.38(6.21$ to 10.56$)$ & $<0.001$ & 97 \\
\hline Anemiał & 7 & 1763 & 0.50 (0.39 to 0.64$)$ & $<0.001$ & 85 \\
\hline Iron deficiency $\ddagger$ & 4 & 1020 & 0.21 (0.07 to 0.63 ) & 0.006 & 85 \\
\hline Ferritin, $g / L \dagger$ & 14 & 3612 & 28.45 (18.03 to 38.86$)$ & $<0.001$ & 98 \\
\hline \multicolumn{6}{|l|}{ Other micronutrients } \\
\hline Serum zinc, $\mathrm{mol} / \mathrm{L} \dagger$ & 5 & 1553 & $0.16(-0.46$ to 0.79$)$ & 0.6 & 76 \\
\hline \multicolumn{6}{|l|}{ Safety } \\
\hline Malaria prevalence $\ddagger$ & 4 & 919 & $1.10(0.94$ to 1.29$)$ & 0.2 & 0 \\
\hline $\begin{array}{l}\text { Rates of positive malaria test } \\
\text { (any species)§ }\end{array}$ & 4 & 1818 & 1.05 (0.90 to 1.21$)$ & 0.6 & 0 \\
\hline Gastrointestinal upsetł & 4 & 576 & 1.30 (0.89 to 1.91$)$ & 0.2 & 0 \\
\hline Constipation $\ddagger$ & 2 & 202 & 3.44 (0.66 to 19.68$)$ & 0.2 & 6 \\
\hline Vomiting $\ddagger$ & 2 & 202 & 0.86 (0.13 to 5.67$)$ & 0.9 & 0 \\
\hline $\begin{array}{l}\text { *Standardized mean difference. } \\
\text { † Mean difference. } \\
\text { †Risk ratio. } \\
\text { §Rate ratio. }\end{array}$ & & & & & \\
\hline
\end{tabular}


adherence rates: Sen and Kanani ${ }^{52-54}$ reported an adherence rate of $72 \%$, and Nchito and colleagues $^{38.40}$ adherence of 50\%. Six studies (2651 children) excluded participants who failed to take a predefined percentage of supplements (ranging from $66 \%$ to $90 \%$ of total tablets)..$^{22,23,44,47-51,55}$ Five studies (1894 children) compared adherence between children taking iron and control; they reported similar proportions of children taking at least a specified percentage of tablets. ${ }^{19,21,25,27,33}$

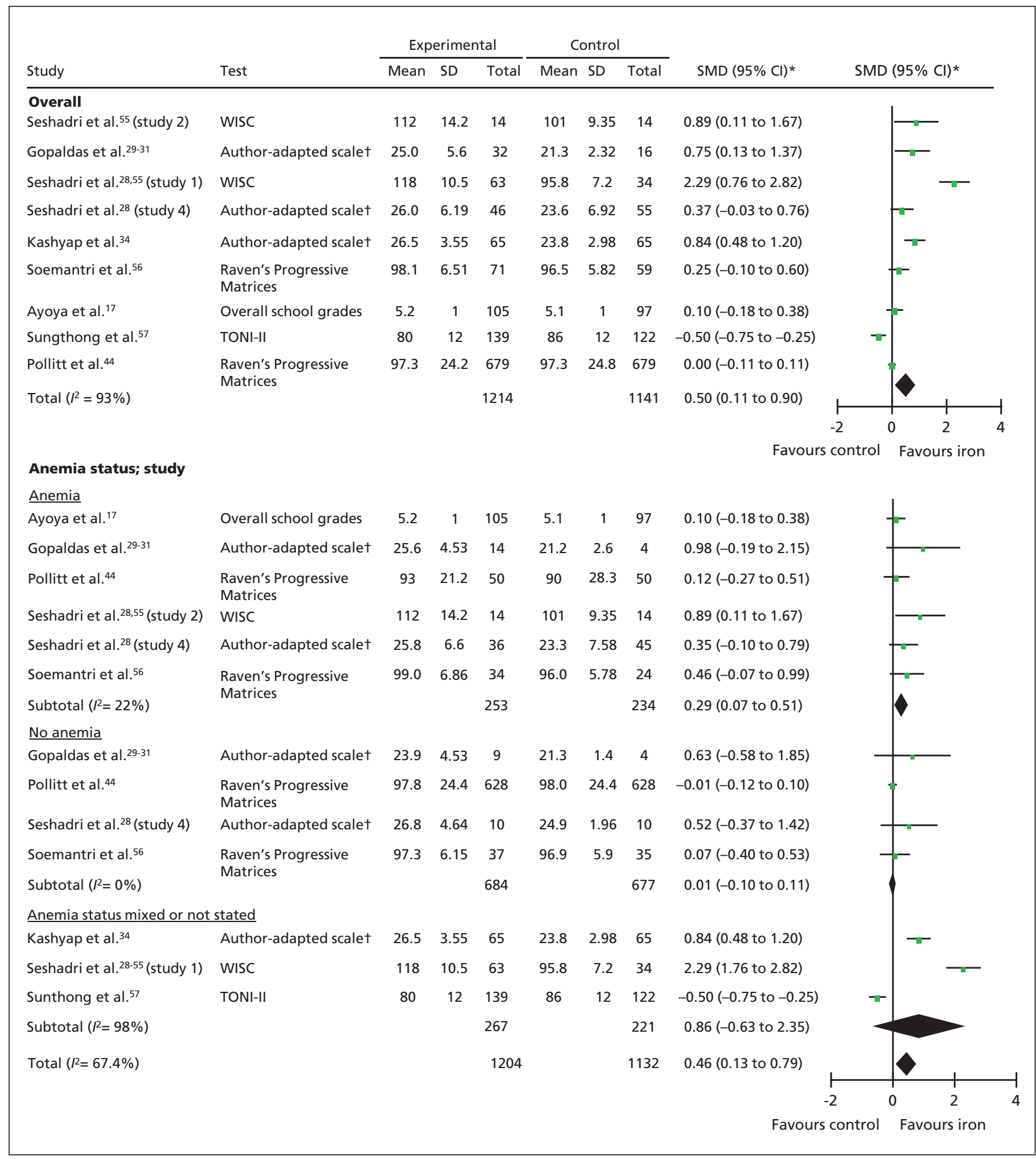

Figure 2: Forest plots for global cognitive performance and cognitive performance by anemia status. *Inverse variance, random effects. tAuthor-adapted scales included clerical task test, visual memory test, digit span test and maze test. Note: $\mathrm{Cl}=\mathrm{Confidence}$ interval, TONI-II = Test of Non-verbal Intelligence, 2nd edition, SD = standard deviation, SMD = standardized mean difference, WISC = Wechsler Intelligence Scale for Children. 


\section{Interpretation}

Daily supplementation provides the highest dose of iron of any nonparenteral approach and is a commonly recommended clinical and public health strategy for the prevention and treatment of anemia. ${ }^{2}$ Potential strategies for anemia control include improved dietary iron by improving dietary diversity or fortification of staple foods and condiments, home-based fortification with multiple micronutrients, iron supplementation and control of helminthic infection. ${ }^{6}$ Our data are chiefly extrapolatable to iron supplementation interventions; alternative approaches to improving iron stores such as food fortification are under evaluation by other systematic reviews. ${ }^{61}$

In this review, we found evidence of a benefit of iron supplementation on cognitive performance among primary-school-aged children, including on IQ among children with anemia. Iron may also improve growth. Daily iron supplementation decreased the prevalence of anemia by about $50 \%$ and reduced the prevalence of iron deficiency by $79 \%$. Iron supplements appear to be well-tolerated, but safety data are limited.

Previous systematic reviews have evaluated the efficacy of iron delivered by multiple approaches (i.e., supplementation, parenteral administration, fortification) among children of broad age ranges; outcomes included hemoglobin level, ${ }^{62}$ mental and physical development, ${ }^{63}$ growth, ${ }^{64}$ exercise capacity, ${ }^{65}$ malaria $^{8}$ and infectious diseases ${ }^{66}$ (but not other adverse effects). Iron supplementation among iron-deficient children without anemia has also been reviewed. ${ }^{67}$ A Cochrane review of intermittent iron supplementation in children found insufficient studies reporting cognitive outcomes or adverse effects. ${ }^{15}$

Two previous reviews have evaluated the effects of iron on cognitive outcomes among primary-school-aged children. Sachdev and colleagues ${ }^{63}$ reported improvements in overall cognitive performance (SMD 0.30) and IQ (SMD 0.41) among children who received parenteral or enteral iron (including fortification). Falkingham and colleagues ${ }^{68}$ found no improvement in overall IQ from iron supplementation, but they reported improvements in IQ among participants with anemia (MD 2.5) and improvements in attention and concentration among adults and children older than 6 years of age.

Our search strategy enabled us to identify and include more eligible studies than either Falkingham and colleagues ${ }^{68}$ (5 additional studies) or Sachdev and colleagues ${ }^{63}$ (7 additional studies). We have improved on previous reviews by performing a broader search, providing a comprehensive evaluation of the benefits and safety, and addressing a specific, widely used strategy of iron administration among children at an age at which developmental benefits are critical.

\section{Limitations}

Our conclusions are limited by poor reporting in the included studies: many studies did not report the randomization method (20 studies), allocation concealment (25 studies) or blinding (18 studies). Although biochemically measured outcomes (e.g., hematologic and iron indices) are unlikely to be affected by the blinding of outcome assessors, cognitive scores have the potential for performance and detection bias.

Our review is also limited by significant het-

\begin{tabular}{|c|c|c|c|c|c|c|c|c|}
\hline \multirow[b]{2}{*}{ Study } & \multicolumn{3}{|c|}{ Experimental } & \multicolumn{3}{|c|}{ Control } & \multirow{2}{*}{$\begin{array}{l}\text { Mean difference } \\
(95 \% \mathrm{Cl})^{*}\end{array}$} & \multirow{2}{*}{$\begin{array}{l}\text { Mean difference } \\
(95 \% \mathrm{Cl})^{*}\end{array}$} \\
\hline & Mean & SD & Total & Mean & SD & Total & & \\
\hline Chwang et al. ${ }^{24}$ & -1.55 & 0.37 & 59 & -1.57 & 0.33 & 60 & $0.02(-0.11$ to 0.15$)$ & \\
\hline Lawless et al. ${ }^{37}$ & -1.24 & 1.06 & 42 & -1.34 & 1.06 & 44 & $0.10(-0.35$ to 0.55$)$ & \\
\hline Perrone et al. ${ }^{42}$ & -2.48 & 0.23 & 8 & -2.70 & 0.12 & 8 & $0.23(0.05$ to 0.41$)$ & - \\
\hline Richard et al. ${ }^{46}$ & -2.04 & 0.85 & 210 & -2.11 & 0.88 & 209 & $0.07(-0.10$ to 0.24$)$ & . \\
\hline $\begin{array}{l}\text { Richard et al. }{ }^{46} \\
\text { (iron alone) }\end{array}$ & -2.05 & 0.89 & 208 & -2.22 & 0.89 & 209 & $0.17(0.00$ to 0.34$)$ & \\
\hline Sungthong et al. ${ }^{57}$ & -1.62 & 0.90 & 139 & -1.59 & 0.90 & 122 & $-0.03(-0.25$ to 0.19$)$ & \\
\hline \multirow[t]{3}{*}{ Total $\left(R^{2}=10 \%\right)$} & & & 666 & & & 652 & 0.09 (0.01 to 0.17$)$ & \\
\hline & & & & & & & & 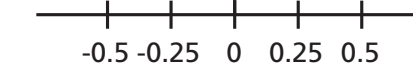 \\
\hline & & & & & & & & Favours control Favours iron \\
\hline
\end{tabular}

Figure 3: Forest plot for height. Forest plot of the z scores for height. *Inverse variance, random effects. Note: $\mathrm{Cl}=$ confidence interval, SD = standard deviation. 
erogeneity, which we addressed by use of random effects meta-analysis and predefined subgroup analyses. This heterogeneity appears to be mainly because of differences in baseline anemia status. Unfortunately, several important outcomes (particularly physical performance and growth) were reported by relatively few studies, and only 6 studies reported safety outcomes. Further randomized trials evaluating the nonhematologic effects of iron, especially outcomes such as cognitive performance, growth and safety, are needed.

\section{Conclusion}

In this review, we found evidence that iron supplementation benefits global cognitive performance. Anemia and iron deficiency were prevalent in the included studies (69\% and 59\%, respectively, in the control groups); thus, routine daily iron supplementation is likely to benefit cognitive performance in primary-school-aged children in developing settings where anemia is prevalent and testing hemoglobin before iron supplementation may not be feasible. In developed settings, our data support ensuring that anemia is detected and treated. Reductions in the prevalence of anemia and iron deficiency reported here should guide the targets of anemia-control programs. Cognitive performance is associated with educational achievement, ${ }^{69}$ future income and productivity. ${ }^{70}$ Daily iron supplementation could benefit educational attainment and economic potential at the individual level and, in settings where anemia is prevalent, population level.

\section{References}

1. Benoist B, McLean E, Egli I, et al. Worldwide prevalence of anaemia 1993-2005. Geneva (Switzerland): World Health Organization; 2008.

2. Iron deficiency: assessment, prevention, and control: a guide for programme managers. Geneva (Switzerland): World Health Organization and United Nations Children's Fund/United Nations University; 2001.

3. Stoltzfus RJ, Chwaya HM, Tielsch JM, et al. Epidemiology of iron deficiency anemia in Zanzibari schoolchildren: the importance of hookworms. Am J Clin Nutr 1997;65:153-9.

4. Christofides A, Schauer C, Zlotkin SH. Iron deficiency and anemia prevalence and associated etiologic risk factors in First Nations and Inuit communities in Northern Ontario and Nunavut. Can J Public Health 2005;96:304-7.

5. Lawson MS, Thomas M, Hardiman A. Iron status of Asian children aged 2 years living in England. Arch Dis Child 1998;78:420-6.

6. Pasricha SR, Drakesmith H, Black J, et al. Control of iron deficiency anemia in low and middle-income countries. Blood 2013; 121:2607-17.

7. Cooper M, Greene-Finestone L, Lowell H, et al. Iron sufficiency of Canadians. Ottawa (ON): Statistics Canada; 2012. Cat. no. 82-003-X

8. Okebe JU, Yahav D, Shbita R.M.P. Oral iron supplements for children in malaria-endemic areas. Cochrane Database Syst Rev 2011;(10)CD006589.

9. Stoltzfus RJ, Mullany L, Black RE. Iron deficiency anaemia. In: Ezzati M, Lopez AD, Rodgers A, et al., editors. Comparative quantification of health risks: global and regional burden of disease attributable to selected major risk factors. Geneva (Switzerland): World Health Organization; 2004.

10. Grantham-McGregor S, Ani C. A review of studies on the effect of iron deficiency on cognitive development in children. $J$ Nutr 2001;131:949S-666S.

11. McCann JC, Ames BN. An overview of evidence for a causal rela- tion between iron deficiency during development and deficits in cognitive or behavioral function. Am J Clin Nutr 2007;85:931-45.

12. Goswami U, Bryant P. Children's cognitive development and learning: primary review research survey 2/1a. Cambridge (UK): University of Cambridge, Faculty of Education; 2007.

13. Pena-Rosas JP, De-Regil LM, Rogers LM, et al. Translating research into action: WHO evidence-informed guidelines for safe and effective micronutrient interventions. $J$ Nutr 2012;142: 197S-204S

14. Higgins JP, Altman DG, Sterne JA. Assessing risk of bias in included studies. In: Higgins JPT, Green S, editors. Cochrane handbook for systematic reviews of interventions. Oxford (UK): The Cochrane Collaboration; 2011.

15. De-Regil LM, Jefferds ME, Sylvetsky AC, et al. Intermittent iron supplementation for improving nutrition and development in children under 12 years of age. Cochrane Database Syst Rev 2011;(12):CD009085.

16. Introduction to meta-analysis. Borenstein M, Hedges L, Rothstein H. Chichester (UK): John Wiley \& Sons; 2007.

17. Ayoya MA, Spiekermann-Brouwer GM, Traoré AK, et al. Effect on school attendance and performance of iron and multiple micronutrients as adjunct to drug treatment of Schistosomainfected anemic schoolchildren. Food Nutr Bull 2012;33:235-41.

18. Ayoya MA,Spiekermann-Brouwer GM, Traoré AK, Stoltzfus RJ, et al. Multiple micronutrients including iron are not more effective than iron alone for improving hemoglobin and iron status of Malian school children. J Nutr 2009;139:1972-9.

19. Berger J, Aguayo VM, Tellez W, et al. Weekly iron supplementation is as effective as 5 day per week iron supplementation in Bolivian school children living at high altitude. Eur J Clin Nutr 1997;51:381-6.

20. Boivin MJ, Giordani B. Improvements in cognitive performance for schoolchildren in Zaire, Africa, following an iron supplement and treatment for intestinal parasites. J Pediatr Psychol 1993;18:249-64.

21. Bradfield RB, Jensen MV, Gonzales L, et al. Effect of low-level iron and vitamin supplementation on a tropical anemia. Am J Clin Nutr 1968;21:57-67.

22. Charoenlarp P. Effect of iron and folate supplementation on haematocrit levels of school children in a rural area of Central Thailand. Southeast Asian J Trop Med Public Health 1973;4:588-92.

23. Charoenlarp P, Pholpothi T, Chatpunyaporn P, et al. The effect of riboflavin on the hematologic changes in iron supplementation of schoolchildren. Southeast Asian J Trop Med Public Health 1980; 11:97-103.

24. Chwang LC, Soemantri AG, Pollitt E. Iron supplementation and physical growth of rural Indonesian children. Am J Clin Nutr 1988; 47:496-501.

25. de Silva A, Atukorala S, Weerasinghe I, et al. Iron supplementation improves iron status and reduces morbidity in children with or without upper respiratory tract infections: a randomized controlled study in Colombo, Sri Lanka. Am J Clin Nutr 2003;77:234-41.

26. Fallahi E, Kimiagar M, Nazari A, et al. Effect of zinc and iron supplementation on indicators of iron, zinc and vitamin A status of primary school children. Pak J Biol Sci 2007;10:1088-92.

27. Gebreselassie H. Iron supplementation and malaria infection: results of a randomized controlled field trial [dissertation]. Montreal (QC): McGill University, 1996.

28. Seshadri S, Gopaldas T. Impact of iron supplementation on cognitive functions in preschool and school-aged children: the Indian experience. Am J Clin Nutr 1989;50:675-84, discussion 85-6.

29. Gopaldas T, Kale M. Prophylactic iron supplementation for underprivileged school boys. I. Two levels of dosing and efficacy of teacher-distributions. Indian Pediatr 1985;22:731-6.

30. Gopaldas T, Kale M, Bhardwaj P. Prophylactic iron supplementation for underprivileged school boys. III. Impact on submaximal work capacity. Indian Pediatr 1985;22:745-52.

31. Gopaldas T, Kale M, Bhardwaj P. Prophylactic iron supplementation for underprivileged school boys. II. Impact on selected tests of cognitive function. Indian Pediatr 1985;22:737-43.

32. Greisen G. Mild anaemia in African school children: effect on running performance and an intervention trial. Acta Paediatr Scand 1986;75:662-7.

33. Harvey PWJ, Heywood PF, Nesheim MC, et al. The effect of iron therapy on malarial infection in Papua New Guinean schoolchildren. Am J Trop Med Hyg 1989;40:12-8.

34. Kashyap P, Gopaldas T. Impact of hematinic supplementation on cognitive function in underprivileged school girls (8-15 yrs of age). Nutr Res 1987;7:1117-26.

35. Konofal E, Lecendreux M, Deron J, et al. Effects of iron supplementation on attention deficit hyperactivity disorder in children. Pediatr Neurol 2008;38:20-6.

36. Latham MC, Stephenson LS, Kinoti SN, et al. Improvements in growth following iron supplementation in young Kenyan school children. Nutrition 1990;6:159-65. 
37. Lawless JW, Latham MC, Stephenson LS, et al. Iron supplementation improves appetite and growth in anemic Kenyan primary school children. J Nutr 1994;124:645-54.

38. Nchito M, Friis H, Michaelsen KF, et al. Iron supplementation increases small intestine permeability in primary schoolchildren in Lusaka, Zambia. Trans R Soc Trop Med Hyg 2006;100:791-4

39. Nchito M, Geissler PW, Mubila L, et al. Effects of iron and multimicronutrient supplementation on geophagy: a two-by-two factorial study among Zambian schoolchildren in Lusaka. Trans $R$ Soc Trop Med Hyg 2004;98:218-27.

40. Nchito M, Geissler PW, Mubila L, et al. The effect of iron and multi-micronutrient supplementation on Ascaris lumbricoides reinfection among Zambian schoolchildren. Trans $R$ Soc Trop Med Hyg 2009;103:229-36.

41. Paracha PI, Khan SM, Ahmad I, et al. Effect of iron supplementation on biochemical indices of iron status in selected preadolescent schoolgirls in North West Frontier Province, Pakistan. Asia Pac J Clin Nutr 1993;2:177-81.

42. Perrone L, Salerno M, Gialanella G, et al. Long-term zinc and iron supplementation in children of short stature: effect of growth and on trace element content in tissues. J Trace Elem Med Biol 1999;13:51-6.

43. Hathirat P, Valyasevi A, Kotchabhakdi NJ, et al. Effects of an iron supplementation trial on the Fe status of Thai schoolchildren. Br J Nutr 1992;68:245-52.

44. Pollitt E, Hathirat $\mathrm{P}$, Kotchabhakdi NJ, et al. Iron deficiency and educational achievement in Thailand. Am J Clin Nutr 1989;50: 687-96, discussion 96-7.

45. Powers HJ, Bates CJ, Prentice AM, et al. The relative effectiveness of iron and iron with riboflavin in correcting a microcytic anaemia in men and children in rural Gambia. Hum Nutr Clin Nutr 1983;37:413-25.

46. Richard SA, Zavaleta N, Caulfield LE, et al. Zinc and iron supplementation and malaria, diarrhea, and respiratory infections in children in the Peruvian Amazon. Am J Trop Med Hyg 2006;75: 126-32.

47. Kordas K. Effects of iron and zinc supplementation on cognitive function, achievement and behavior of lead-exposed Mexican school children. Baltimore (MD): Johns Hopkins University; 2003.

48. Kordas K, Stoltzfus RJ, Lopez P, et al. Iron and zinc supplementation does not improve parent or teacher ratings of behavior in first grade Mexican children exposed to lead. J Pediatr 2005; 147:632-9.

49. Rico JA, Kordas K, Lopez P, et al. Efficacy of iron and/or zinc supplementation on cognitive performance of lead-exposed Mexican schoolchildren: a randomized, placebo-controlled trial. Pediatrics 2006;117:e518-27

50. Rosado JL, Lopez P, Kordas K, et al. Iron and/or zinc supplementation did not reduce blood lead concentrations in children in a randomized, placebo-controlled trial. J Nutr 2006;136:2378-83.

51. Rosales FJ, Kang Y, Pfeiffer B, et al. Twice the recommended daily allowance of iron is associated with an increase in plasma alpha-1 antichymotrypsin concentrations in Guatemalan schoolaged children. Nutr Res 2004; 24:875-87.

52. Sen A, Kanani SJ. Impact of iron-folic acid supplementation on cognitive abilities of school girls in Vadodara. Indian Pediatr 2009;46:137-43.

53. Sen A, Kanani SJ. Physical work capacity of young underprivileged school girls impact of daily vs intermittent iron folic acid supplementation: a randomized controlled trial. Indian Pediatr 2009;46:849-54

54. Sen A, Kanani S. Intermittent iron folate supplementation: impact on hematinic status and growth of school girls. ISRN Hematol 2012:482153.

55. Seshadri S, Hirode K, Naik P, et al. Behavioural responses of young anaemic Indian children to iron-folic acid supplements. Br J Nutr 1982;48:233-40.

56. Soemantri AG. Preliminary findings on iron supplementation and learning achievement of rural Indonesian children. Am J Clin Nutr 1989;50:698-701; discussion -2.

57. Sungthong R, Mo-Suwan L, Chongsuvivatwong V, et al. Once weekly is superior to daily iron supplementation on height gain but not on hematological improvement among schoolchildren in Thailand. J Nutr 2002;132:418-22.

58. Sungthong R, Mo-suwan L, Chongsuvivatwong V, et al. Onceweekly and 5-days a week iron supplementation differentially affect cognitive function but not school performance in Thai children. J Nutr 2004;134:2349-54.

59. Hedges L, Hedberg EC. Intraclass correlations for planning group randomized experiments in rural education. J Res Rural Educ $2007 ; 22$.
60. Kriemler S, Schindler C, Meyer U, et al. Effect of school based physical activity programme (KISS) on fitness and adiposity in primary schoolchildren: cluster randomized controlled trial. BMJ 2010;340:c785

61. Pasricha S-R, De-Regil LM, Garcia-Casal MN, et al. Fortification of maize flour with iron for preventing anaemia and iron deficiency in populations. Cochrane Database Syst Rev 2012;(11)CD010187.

62. Gera T, Sachdev HP, Nestel P, et al. Effect of iron supplementation on haemoglobin response in children: systematic review of randomised controlled trials. J Pediatr Gastroenterol Nutr 2007; 44:468-86.

63. Sachdev H, Gera T, Nestal P. Effect of iron supplementation on mental and motor development in children: systematic review of randomized controlled trials. Public Health Nutr 2005;8:117-32.

64. Sachdev H, Gera T, Nestel P. Effect of iron supplementation on physical growth in children: systematic review of randomized controlled trials. Public Health Nutr 2006;9:904-20.

65. Gera T, Sachdev S, Nestal P. Effect of iron supplementation on physical performance in children and adolescents: systematic review of randomized controlled trials. Indian Pediatr 2007;44: 15-24.

66. Gera T, Sachdev HP. Effect of iron supplementation on incidence of infectious illness in children: systematic review. $B M J$ 2002;325:1142.

67. Abdullah K, Kendzerska T, Shah P, et al. Efficacy of oral iron therapy in improving the developmental outcome of pre-school children with non-anaemic iron deficiency: a systematic review. Public Health Nutr 2013;16:1497-506.

68. Falkingham M, Abdelhamid A, Curtis P, et al. The effects of oral iron supplementation on cognition in older children and adults: a systematic review and meta-analysis. Nutr J 2010;9:4.

69. Taubman P, Wales T. Mental ability and higher education attainment in the 20th century. New York (NY): McGraw-Hill; 1972.

70. Horton S, Ross J. The economics of iron deficiency. Food Policy $2003 ; 28: 51-75$.

Affiliations: Department of Clinical Haematology (Low), The Alfred Hospital, Prahran; Thalassaemia Service (Farrell, Pasricha), Southern Health, Clayton; Department of Medicine (Biggs, Pasricha), The Royal Melbourne Hospital, Faculty of Medicine, Dentistry and Health Sciences, The University of Melbourne, Carlton; Nossal Institute for Global Health (Pasricha), Faculty of Medicine, Dentistry and Health Sciences, The University of Melbourne, Carlton, Victoria, Australia.

Contributors: Sant-Rayn Pasricha and Michael Low conceived the systematic review design, developed the search strategy, undertook the search, screened papers for eligibility, extracted data, analyzed the results and wrote the manuscript. Ann Farrell screened papers for eligibility, extracted data and wrote the manuscript. Beverley-Ann Biggs conceived the systematic review design, interpreted the data and revised the manuscript critically for important intellectual content. All of the authors approved the final version submitted for publication.

Funding: Sant-Rayn Pasricha was supported by a Victoria Fellowship from the Government of Victoria; a CRB Blackburn Scholarship from the Royal Australasian College of Physicians; an Overseas Research Experience Scholarship from the University of Melbourne; and a National Health and Medical Research Council CJ Martin Early Career Fellowship. Michael Low was supported by an Alfred Research Trust Grant. The funders had no role in study design, data collection and analysis, decision to publish, or preparation of the manuscript.

Acknowledgements: The authors thank the staff in the Department of Nutrition for Health and Development and the Library and Information Networks for Knowledge, World Health Organization, Geneva, for their guidance and assistance in planning and implementing this study. They also thank Patrick Condron, librarian at The University of Melbourne, for assistance with the electronic search strategies, and Maggie Low, University of Adelaide, and Dr. Denise Lee, Alfred Hospital, for assistance with acquiring the publications. 